\title{
Reynolds Pressure and Relaxation in a Sheared Granular System
}

\author{
Jie Ren, ${ }^{1}$ Joshua A. Dijksman, ${ }^{1}$ and Robert P. Behringer ${ }^{1}$ \\ ${ }^{1}$ Department of Physics $\&$ Center for Non-linear and Complex Systems, \\ Duke University, Science Drive, Durham NC 27708-0305, USA
}

(Dated: January 22, 2018)

\begin{abstract}
We describe experiments that probe the evolution of shear jammed states, occurring for packing fractions $\phi_{S} \leq \phi \leq \phi_{J}$, for frictional granular disks, where above $\phi_{J}$ there are no stress-free static states. We use a novel shear apparatus that avoids the formation of inhomogeneities known as shear bands. This fixed $\phi$ system exhibits coupling between the shear strain, $\gamma$, and the pressure, $P$, which we characterize by the 'Reynolds pressure', and a 'Reynolds coefficient', $R(\phi)=\left(\partial^{2} P / \partial \gamma^{2}\right) / 2$. $R$ depends only on $\phi$, and diverges as $R \sim\left(\phi_{c}-\phi\right)^{\alpha}$, where $\phi_{c} \simeq \phi_{J}$, and $\alpha \simeq-3.3$. Under cyclic shear, this system evolves logarithmically slowly towards limit cycle dynamics, which we characterize in terms of pressure relaxation at cycle $n: \Delta P \simeq-\beta \ln \left(n / n_{0}\right) . \quad \beta$ depends only on the shear cycle amplitude, suggesting an activated process where $\beta$ plays a temperature-like role.
\end{abstract}

PACS numbers: 83.80.Fg, 62.20.D-, 83.85.Vb

Keywords: Granular materials, jamming, shear jamming

Much recent work has focused on the mechanical behavior of disordered solids, including granular materials, colloids, foams and molecular glass formers. These systems are well known for their glassy flow behavior and surprising rigidity. Notably, Bi et al. 4, 5] recently showed that in frictional systems, e.g. most common granular materials, shear strain, $\gamma$, can 'shear jam 5. a loose, low density packing of particles, enabling it to support a shear stress. The nature of these shear jammed states, particularly how they form and evolve, is an unsolved problem with obvious relevance, whose understanding is the goal of the current paper.

To set the context, we note that $\mathrm{Bi}$ et al. [5] showed that there is a lowest packing fraction $\phi_{J}$, such that below (above) this density, there are (no) zero-stress states. Application of shear to a zero-stress state in $\phi_{S} \leq \phi \leq \phi_{J}$ leads to highly anisotropic contact and force networks, and to non-zero shear stress, $\tau$, and pressure, $P$. Here, $\tau=\left(\sigma_{1}-\sigma_{2}\right) / 2$ and $P=\left(\sigma_{1}+\sigma_{2}\right) / 2$, where the $\sigma_{i}$ are the principal stresses of the 2D stress tensor, $\hat{\sigma}$. Starting from zero stress, the system traverses a fragile regime, and with additional shear strain, the system arrives at a fully jammed state where the force/contact networks percolate in all directions. These shear jammed states may occur naturally in many granular systems, such as geophysical flows, sand and suspensions. Improved understanding of shear jammed states is thus crucial for both a better understanding of the concept of jamming for (frictional) materials, and to shed light on the complex rheology of dense granular media [6].

At the heart of shear jamming are classic studies by Reynolds, who showed that under fixed pressure, granular systems can dilate in response to shear [7]. Despite its relevance, a quantitative understanding of this effect has remained elusive over the last century. This is partly due to a complication in the study of sheared frictional materials: Shear typically induces the formation of dilated localized shear bands, where most of the shear strain is confined. System-wide measures may tend to reflect the band properties rather than the whole system, making it difficult to interpret experiments.

To understand the important physics underlying shear jamming, it is crucial to have an experimental approach that avoids shear banding. In this Letter, we describe such an approach that, for the first time to our knowledge, avoids shear banding. Measurements using this method provide the first characterizations of, and key insights into, the mechanical response and dynamics of shear jammed frictional packings. In these fixed volume experiments, the response to shear is manifested as a nonlinearly growing pressure with shear strain, which is related to Reynolds' dilatancy. Associated with this pressure effect are structural rearrangements that lead to a surprising Arrhenius-like stress relaxation dynamics in periodically sheared disk packings.

Key Findings In these experiments, we shear a disordered disk packing (2D) at fixed density. In such a system, dilatancy cannot occur, but a related phenomena occurs: the stresses $\hat{\sigma}$ respond to the shear strain. We find that $P$ increases roughly as $\gamma^{2}$, which we describe by a "Reynolds coefficient", $R=\left(\partial^{2} P / \partial \gamma_{\mid \phi}^{2}\right) / 2$. We find that $R$ depends only on $\phi$, and it provides a simple parametrization of the coupling between $P$ and $\gamma . R$ seems to diverge as $\phi$ approaches $\phi_{c} \simeq \phi_{J}$, thus identifying a special role for $\phi_{J}$ for the shear jamming states.

An additional key observation from this work is that for $\phi_{S} \leq \phi \leq \phi_{J}$ the stress response to cyclic shear strain shows slow relaxational dynamics to a limit cycle, that depends on driving. The deviation from a limit cycle, measured by pressure, shows a logarithmic decay over time/cycle number. The data for stress relaxation exhibit a totally unexpected scaling form, as developed below.

Experimental Setup Key to these experiments is a novel 


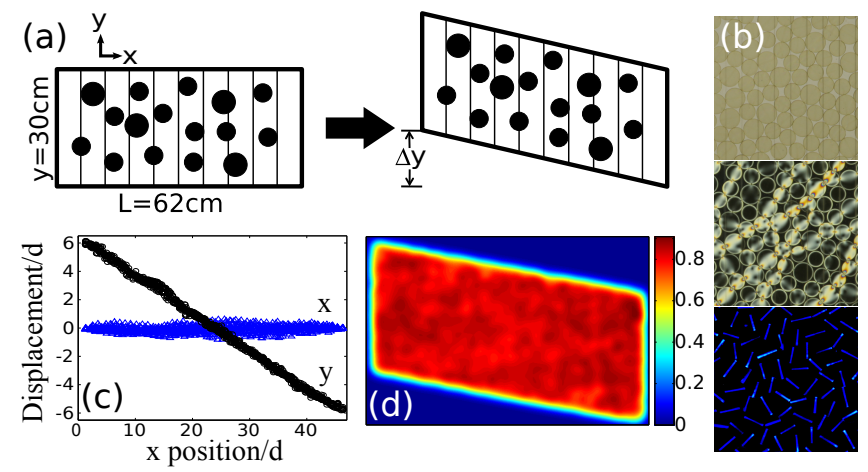

FIG. 1: (Color online) (a) Setup schematics (b) The three close-up images that the camera captures at each step: particle positions (upper), force response under polariscope (middle), and particle orientation images under UV light (lower). (c) The $x$ - and $y$-displacement of particles vs. their horizontal positions in the system, and (d) the coarse grained [15, 16] density profile after $27 \%$ linear shear.

apparatus that provides (simple) shear throughout the system, in contrast to wall-driven shear. The base of the apparatus consists of narrow, parallel, horizontal, and transparent slats. Shear is applied by deforming the slats and boundary uniformly in the ' $y$ ' direction, keeping the ' $\mathrm{x}$ ' dimension fixed at $L$, to provide uniform simple shear strain $\gamma=\Delta y / L$ at constant packing fraction $\phi$ (Fig. 17a). On the slats rest $\sim 1000$ bi-disperse photo-elastic particles (Vishay PSM-4) of diameters $12.7 \mathrm{~mm}$ and $15.9 \mathrm{~mm}$; the slat width is of the order of the particle size. The relative numbers of large to small particles is set to $1: 3.3$, in order to prevent crystallization. Before each experiment, we prepare a stress-free packing by rearranging the particles (gently tapping or pushing particles) until no visual photoelastic response is visible. This bottomassisted shear induces a linear shear profile, suppressing shear bands and the usual inhomogeneities. It is reminiscent of the SLLOD and related algorithms 8 for enforcing uniform shear in MD simulations. It bears some resemblance to 3D experiments by Mueggenburg [9], but with a key difference: in the Mueggenburg experiments, a slat geometry was used, but the slot motion was not coordinated, and sustained uniform shear did not occur. We note that a small background pressure of $\sim 0.5 \mathrm{~N} / \mathrm{m}$ is detected, even in the absence of shear. This is due in roughly equal amounts to small experimental errors in force determinations, our ability to completely relax all inter-particle forces, and weak friction between the particles and the slats.

The experiment is illuminated from below by circularly polarized uniform white light, and from above by a less intense UV light. A 22 Megapixel camera above the experiment records views with and without a circular polarizer. We apply quasi-static shear strain in small steps. After each step, we pause and record three views of the system which respectively yield particle positions, photo- elastic responses, and rotations. Without crossed polarizers, the edges of the particles are visible (Fig.1p upper), and we use a circular Hough-transform technique [10] to determine particle centers with an accuracy of $\sim 0.02 d$. With a circular polarizer in front of the camera, we image the photo-elastic pattern of colored fringes within each particle, which encode the contact forces acting on each particle (Fig. 1 $1 \mathrm{p}$ middle). To determine the particle orientations, each disk is marked diametrically with a line of fluorescent dye, visible under UV light with the white light turned off (Fig. 1p lower). Changes in the bar orientations give particle rotations. The complete process of multiple strain steps, followed by imaging after each step, is fully automated, and we record up to 500 shear cycles per run. We extract the local particle stress by either a pattern-fitting approach [4, 11, yielding the complete contact network, particle forces, and stress tensor (e.g. $P$ and $\tau$ ), or via $G^{2}$, the local squared intensity gradient of the photo-elastic response, averaged on each particle [12, 13]. $G^{2}$ is a one-to-one function of $P$ on the particle level, providing an efficient measure for $P$. For small/large data sets, we use the former/latter approach.

Reynolds Effect As noted, a striking aspect of applying shear strain to a stress-free state for $\phi_{S} \leq \phi \leq \phi_{J}$, is the generation of nonzero $P$ and $\tau$, as in the shear jamming experiments of $\mathrm{Bi}$ et al. [5]. In the present experiments, we go well beyond $\mathrm{Bi}$ et al. to probe the evolution of shear jammed states, first by forward-shearing the system, and then by shearing cyclically. Regarding forward shear, we prepared packings in a stress free initial state, for $0.691 \leq \phi \leq 0.816$, where $\phi_{J}=0.835 \pm 0.005$, and $\phi_{S} \approx 0.75$. We then quasi-statically shear the system by 200 small strain steps of $0.27 \%$, up to a total strain of $\gamma=54 \%$ [14. These experiments show shear jamming [5], as expected, but unlike previous experiments, particle tracking data (Fig. 1. ) show that the shear is effectively linear and homogeneous across the entire system. Particle displacements and rotations relative to the uniform shear background are small. The locally coarse-grained density field 15, 16, (Fig. 11), shows no sign of a shear band or permanent inhomogeneities.

For the larger $\phi$ 's considered here, we could not apply the full $54 \%$ strain because $P$ became so large that the layer was unstable to out-of-plane buckling. If buckling occurred, we terminated the forward shear experiment. The forward shear results, Fig. 2 a, indicate that the shear-induced 'Reynolds pressure' increases roughly as $\gamma^{2}$ with a density dependent prefactor which we characterize by the 'Reynolds coefficient',

$$
R=\left(\partial^{2} P / \partial \gamma_{\mid \phi}^{2}\right) / 2
$$

For linear isotropic elastic materials, no coupling between shear strain and pressure is expected. But, as we apply shear, the system becomes increasingly anisotropic, so a $P-\gamma$ coupling might be possible, as expressed by, $\partial P / \partial \gamma$. In our system, this derivative grows roughly as 

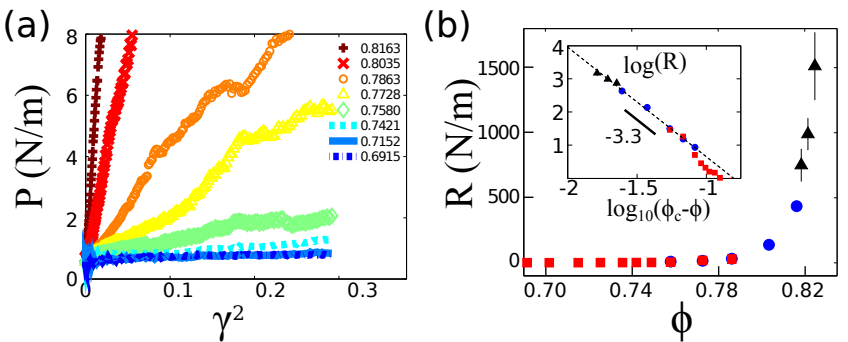

FIG. 2: (Color online) (a) Reynolds pressure $P\left(\gamma^{2}\right)$ observed in forward shear (see text) tests for $\phi=0.691-0.816$. (b) Reynolds coefficient $\mathrm{R}$ extracted from linear fitting, obtained from up to $54 \%$ forward shear (red squares), up to $27 \%$ forward shear (blue dots), and cyclic shear tests under limit cycle behavior (black triangles). The inset shows the same data on double logarithmic scales with $\phi_{c}=0.841 \pm 0.004$. The error bar is smaller than the size of the symbols unless marked. The dashed line shows a fit to a power law. A line corresponding to an exponent -3.3 is also shown for reference.

$\gamma$, and linear elasticity is not a particularly useful concept. $R$ grows strongly with $\phi$, and shows an apparent, but unexpected divergence at $\phi=\phi_{c} \simeq \phi_{J}$. Fig. $2 \mathrm{~b}$ and inset, show a log-log plot of $R$ vs. $\Delta \phi=\phi_{c}-\phi$. A power-law fit to $R=A\left(\phi_{c}-\phi\right)^{\alpha}$, yields $\alpha=-3.3 \pm 0.1$ and $\phi_{c}=0.841 \pm 0.004$. By contrast, $\phi_{c}$ lies in the range $0.83 \leq \phi_{j} \leq 0.84$, so here, $\phi_{c}$ is not distinguishable from $\phi_{J}$, which is also comparable to $\phi_{J}$ for systems of frictionless $2 \mathrm{D}$ particles. For $\phi \leq 0.75$, the system is very loose, and it does not form a percolating contact network, even after $54 \%$ strain. $R(\phi)$ behavior in this case is affected by small experimental 'noise' effects, discussed above, and deviates from the power-law behavior (Fig. $2 \mathrm{~b}$ (inset)). We identify $\phi_{S} \simeq 0.75$, the lower limit in this system for shear jamming.

Limit Cycles To characterize the evolution/reproducibility/relaxation of the stresses, we carried out multiple shear cycles. This also allowed us to determine $R$ for $\phi$ closer to $\phi_{J}$, where shear strains are limited due to buckling; we obtain good statistics by many smaller-amplitude strain cycles. The oscillatory shear experiments were started from initially stress-free states for $\phi$ 's in the shear jamming regime, $\phi_{S} \leq \phi \leq \phi_{J}$. In a cycle, we sheared by strain steps of $0.45 \%$ up to $\gamma_{\max }$ in the 'forward direction', followed by a shear strain decrease $(-0.45 \%$ per step) to a smaller strain, $\gamma_{\min }$. For symmetric shear cycles: $\gamma_{\min }=-\gamma_{\max }$, and asymmetric shear cycles: $\gamma_{\min } \neq-\gamma_{\max }$.

For symmetric cycles, $P$ was symmetric about $\gamma=0$, approximately quadratic in $\gamma$, and virtually reproducible over many cycles, as shown in Fig. 3a. However, details of the network were generally not reproducible from cycle to cycle. The Reynolds coefficient $R(\phi)$ followed the same trend as in the forward shear tests (Fig. 2b), further confirming the Reynolds effect. After transients, the shear stress $\tau$ also followed a reproducible path over cycles, but
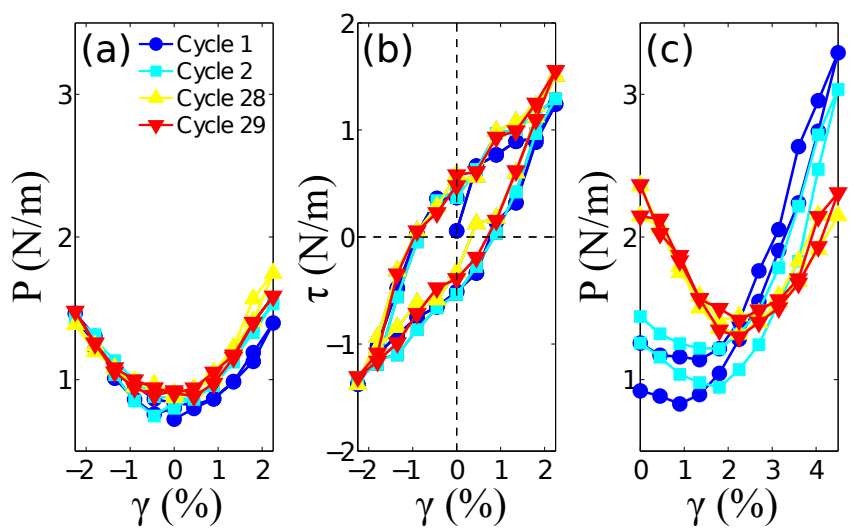

FIG. 3: (Color online) (a) $P$ vs. $\gamma$ for a symmetric cyclic shear run with $\phi=0.825$, which started from $\gamma=0$, and sheared between $\gamma_{\max }=2.25 \%$ and $\gamma_{\min }=-2.25 \%$. Only cycles 1, 2, 28 and 29 are shown in the plot. (b) $\tau$ vs. $\gamma$ for the same run and the same shear cycles. (c) $P$ vs. $\gamma$ at cycles 1 , $2,28,29$ for a non-symmetric cyclic shear run $\left(\gamma_{\max }=4.5 \%\right.$, $\left.\gamma_{\min }=0\right)$ with the same density.

unlike $P, \tau$ was strongly hysteretic, with non-zero values at $\gamma=0$. There were $\gamma$ 's for which $\tau=0$ but $P \neq 0$, for example, in Fig. 3 and b, at $\gamma \approx 1 \%$. However, in such cases, $\tau$ coarse grained at smaller scales than the system size was locally non-zero, even though the global $\tau$ was 0 (e.g. because of spatial variations of the principal stress orientations). Due to length limitations, we consider only the dynamics exhibited by $P$, and we will present the full stress dynamics elsewhere.

The evolution of $P(\gamma)$ for asymmetric shear cycles differed from the symmetric case. Here, $P(\gamma)$ was initially asymmetric, but evolved towards a symmetric shape centered around the mean strain, $\bar{\gamma}$, after many cycles. Thus, the long term $P-\gamma$ dynamics was a limit cycle. The system relaxed quickly (slowly) to the limit cycle if sheared symmetrically (asymmetrically). Fig. 3r shows an example of slow evolution, where a limit cycle was reached after about 28 cycles. In this case $P(\gamma)$ evolved to a symmetric shape, similar to the forward shear experiment, except for a shift; i.e., the system did not reach a completely stress-free state at the mid-point of strain. However, a long term limit cycle was still reached with the same Reynolds coefficient for the given density, $\phi=0.825$.

Slow Relaxation For asymmetric strain cycles, $\Delta P(n)=P\left(\gamma_{\max }\right)-P\left(\gamma_{\min }\right)$ was initially nonzero, but it decreased and ultimately vanished, within fluctuations, for $n=n_{0}$. When the limit cycle was reached, $P$ was symmetric about $\bar{\gamma}=\left(\gamma_{\max }+\gamma_{\min }\right) / 2$. The slow relaxation of $\Delta P$ for asymmetric shear shows striking and novel scaling behavior, which we characterize in terms of $\phi, \bar{\gamma}$ and the shear amplitude $\gamma_{A}$. Experiments to characterize this relaxation spanned $\phi$ 's from above $\phi_{S}$ to just below isotropic jamming $\phi_{J}: 0.780 \leq \phi \leq 0.828$, 

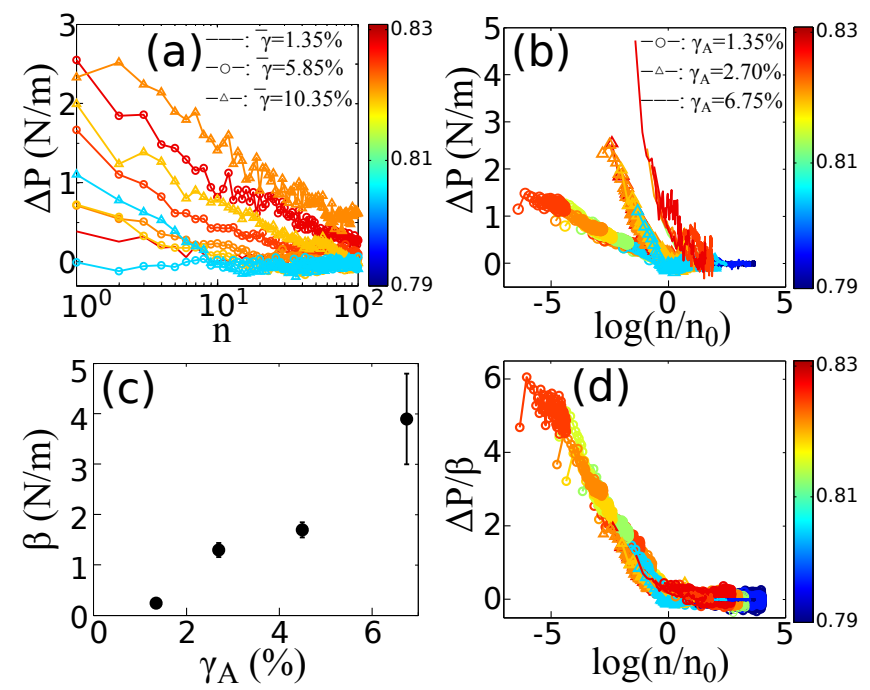

FIG. 4: (Color online) (a) $\Delta P$ vs. number of shear cycles, for various runs with $\gamma_{A}=2.7 \%$, and $\bar{\gamma}=1.35 \%$ (solid line), $5.85 \%$ (open circles), and $10.35 \%$ (open triangles). Different color indicates different $\phi$. (b) $\Delta P$ vs. $\log \left(n / n_{0}\right)$ for 3 different $\gamma_{A}$ 's: $1.35 \%$ (open circles), $2.70 \%$ (open triangles), and $6.75 \%$ (solid lines). Data comes from runs with various $\phi$ (shown by color) and various $\bar{\gamma}$ (not shown). (c) The decay factor, $\beta$, shows a strong increase with $\gamma_{A}$. (d) The universal decay curve, $\Delta P / \beta\left(\gamma_{A}\right)$ vs. $\log \left(n / n_{0}\right)$, including all data from panel (b).

strain amplitudes of $\gamma_{A}=6.75,4.5,3,1.5 \%$ and a range of starting strains $0 \leq \bar{\gamma} \leq 21.35 \%$. Experiments were 100 500 cycles long; for convenience we measured $G^{2}$ only at $\gamma_{\max }, \gamma_{\min }$, and then converted $G^{2}$ to $\Delta P$ using a calibration. Fig. 4 a shows $\Delta P$ for a particular $\gamma_{A}$.

For $\phi$ in the shear jamming region, $\Delta P(n)$ decayed logarithmically slowly towards 0 :

$$
\Delta P(n) \simeq-\beta \log \left(n / n_{0}\right),
$$

implying a natural 'time scale' for relaxation, $n_{0}$, that we obtained through least squares fits of the logarithmic part of the relaxation. All the relaxation data, for a given $\gamma_{A}$, collapse onto a single curve when expressed in terms of $n / n_{0}$ (Fig. 4 4 ), regardless of $\phi$ and $\bar{\gamma}$. The factor $\beta\left(\gamma_{A}\right)$ differs for each $\gamma_{A}$ (Fig. 4 ), but $\Delta P / \beta$ is a universal function of $n / n_{0}$, as in Fig. 4 d, which shows all $\sim 170$ datasets. We emphasize the remarkable role that $\beta\left(\gamma_{A}\right)$ plays, and the fact that it is independent of $\phi$.

We then consider what determines $n_{0}$. Eq. 2 implies: $n_{0}=n \cdot \exp \left(\Delta P(n) / \beta\left(\gamma_{A}\right)\right)$. Initially, at $n=1, \Delta P=$ $\Delta P_{0}$. According to the approximately quadratic relation between $P$ and $\gamma, \Delta P_{0}$ is given by: $\Delta P_{0}=R(\phi)\left(\gamma_{\max }^{2}-\right.$ $\left.\gamma_{\text {min }}^{2}\right) / 2=R(\phi) \bar{\gamma} \gamma_{A}$. Therefore,

$$
n_{0}=\exp \left(R(\phi) \bar{\gamma} \frac{\gamma_{A}}{\beta\left(\gamma_{A}\right)}\right) .
$$

Eq. 2 also implies an evolution $d \Delta P / d n=$ $-\beta n_{0}^{-\mathrm{T}} \exp (\Delta P / \beta)$ or, with a cutoff, $d \Delta P / d n=$ $-\beta n_{0}^{-1}[\exp (\Delta P / \beta)-1]$, which produces the logarithmic form of Eq. 2 for small $n$, with saturation at $n=n_{0}$. This suggests an activated process, perhaps involving a generalized ensemble, such as the stress ensemble, as discussed by several authors 17,20 .

To summarize: for frictional granular systems in/near the shear jamming regime, $\phi_{S} \leq \phi \leq \phi_{J}$, we generated sheared states without shear bands, even with large strains or over many cycles of shear, making it possible to experimentally probe the constitutive relations of granular materials. These experiments show two key and highly novel results: 1) We find a novel Reynolds effect for fixed $\phi$ that is approximately quadratic in $\gamma$ using $R=\left(\partial^{2} P / \partial \gamma_{\mid \phi}^{2}\right) / 2$. We note that the specific form for $R(\gamma)$ may well depend on the particle interaction force; a more general form might be $P=R \gamma^{\delta}$, where for our experiments, $\delta \simeq 2$. 2) We find that under cyclic shear, frictional granular systems evolve logarithmically slowly, as one might expect for an activated process, towards a state where the pressure is symmetric, modulo fluctuations, about the mid-point of strain. The pressure at the symmetry point may not be zero. This slow evolution is characterized by highly novel scaling behavior, such that there is good collapse of all data.

These results point towards several interesting directions. First, it is reasonable to search for a description of these states in terms of an ensemble picture, such as the stress ensemble, given the activated process character of the slow relaxation. Such a theory would need to explain some of the striking scaling properties observed here. In addition, we have not considered the properties of the shear stress under cyclic shearing, nor have we considered the particle dynamics of details of the force/contact networks. We will present these results elsewhere.

We thank Jie Zhang for sharing code to perform rotational particle tracking. IGUS generously supplied us with a free linear stage under the Young Engineers Support program. Discussions with Dapeng Bi, Bulbul Chakraborty, Martin van Hecke, Stefan Luding and Corey O'Hern are gratefully acknowledged. Work supported by NSF grants DMR-0906908, DMR1206351, ARO grant W911NF-11-1-0110, and NSF grant DMS0835742.

[1] A. J. Liu and S. R. Nagel, Nature 396 21-22 (1998).

[2] M. van Hecke, J. Phys. Cond. Matt. 22033101 (2010).

[3] S. Henkes and B. Chakraborty, Phys. Rev. E 79061301 (2009).

[4] J. Zhang, T. Majmudar, A. Tordesillas, and R. P. Behringer, Granular Matter 12 159-172 (2010).

[5] D. Bi, J. Zhang, B. Chakraborty and R. P. Behringer, Nature 480 355-358 (2011).

[6] Y. Forterre and O. Pouliquen, Annu. Rev. Fluid Mech. 40 1-24 (2008). 
[7] O. Reynolds, Phil. Mag. 20 469-481 (1885).

[8] D. J. Evans and G. P. Morriss, Phys. Rev. A 301528 (1984); P. Olsson and S. Teitel, Phys. Rev. Lett. 99 178001 (2007); D. J. Durian, Phys. Rev. Lett. 75 4780$4783(1995)$.

[9] N.W. Mueggenburg, Phys. Rev. E 71031301 (2005).

[10] T. Peng, A. Balijepalli, S. K. Gupta, T. LeBrun, J. Comput. Inf. Sci. Eng. 7330 (2007).

[11] T. S. Majmudar, M. Sperl, S. Luding and R. P. Behringer, Phys. Rev. Lett. 98, 058001 (2007).

[12] D. Howell, R. P. Behringer, and C. Veje, Phys. Rev. Lett. 82 5241-5244 (1999).

[13] Junfei Geng, D. Howell, E. Longhi, R. P. Behringer, G. Reydellet, L. Vanel, E. Clément, and S. Luding, Phys. Rev. Lett. 87035506 (2001).

[14] J. Ren, J. A. Dijksman, and R. P. Behringer, Chaos 21 041105 (2011).
[15] J. Zhang, R. P. Behringer and I. Goldhirsch, Prog. Theor. Phys. Suppl. 184 16-30 (2010).

[16] A. H. Clark, P. Mort and R. P. Behringer, Granular Matter 14 283-288 (2012).

[17] K. A. Reddy, Y. Forterre, and O. Pouliquen, Phys. Rev. Lett. 106108301 (2011).

[18] R. P. Behringer, D. Bi, B. Chakraborty, S. Henkes, and R. R. Hartley, Phys. Rev. Lett. 101 268301(2008).

[19] S. Henkes and B. Chakraborty, Phys. Rev. Lett. 95 198002 (2005); S. Henkes, C. S. O'Hern, and B. Chakraborty, Phys. Rev. Lett. 99038002 (2007).

[20] P. Sollich, F. Lequeux, P. Hébraud, and M. E. Cates, Phys. Rev. Lett. 782020 (1997); P. Sollich, Phys. Rev. E 58738 (1998); P. Sollich and M. E. Cates, Phys. Rev. E 85031127 (2012). 